\title{
Hand Motion Controlled Robotic Arm based on Micro- Electro-Mechanical-System Sensors: Gyroscope, Accelerometer and Magnetometer
}

\author{
Auday A. H. Mohamad \\ Al-Mansour University College \\ Baghdad-Iraq
}

\author{
Basil Tareq Abdulbaqi \\ Al-Mansour University College \\ Baghdad-Iraq
}

\author{
Noor Kareem Jumaa \\ Al-Mansour University College \\ Baghdad-Iraq
}

\begin{abstract}
There are high requirements to develop artificial arms for many inhuman situations where human interactions are presenting challenges or not possible (i.e. impossible situations). This paper presents information, methods, and techniques which are essential for building a robotic arm controlled by the movements of normal human arm (Gesture Robotic Arm) whose data is acquiring by using the sensor fusion technique of Gyroscope, Accelerometers and Magneto sensors (MEMS sensors). For appropriate control mechanism and for reducing the noise amount which is coming in from the sensors, a proper averaging algorithm is used for smoothening the accelerometer output. The development of this arm is based on the Arduino platform; in which all are interfaced with each other by using Bluetooth wireless communication. The prototype of robotic arm of this paper has been implemented practically. The developed robotic arm of this paper is tracked the movement of human arm with a good accuracy. Implementation of this arm could be expected for overcoming the problems such as picking or placing object that are away from the users or could be used in medical applications.
\end{abstract}

\section{Keywords}

MEMS Sensors, Sensor Fusion, Robotic Arm, Gesture Controlled.

\section{INTRODUCTION}

Orientation tracking and human hand orientation has an important role in medical and military applications. Inertial sensors are a technology may be used for observed the orientation of rigid bodies so they are used in real-time motion tracking systems to track the location of the rigid body. Inertial sensors have a small size and low weight and they used to detect physical quantities of the moving object regardless of external references, environment lighting or friction. "Micro-Electro-Mechanical-System (MEMS) based inertial sensors be a popular type of sensors in many fields and applications such as wearable application for detection and tracking due to their miniature size, low power consumption, and light weight". [1,3]

"There are many handy controlled robotic arms named gesture robotic arm with different sensing technologies such as Vision-based Gesture Recognition , Motion Capture Sensor Recognition, Finger Gesture Recognition System based on Active Tracking Mechanisms and Accelerometer-based Gesture Recognition" [1]. These methods gave acceptable results in indoor environments; some constraints make these methods be unacceptable like shadows, distance and interference.
The rest of this paper is organized as following: section II presents the related works, Preliminaries of system components is described in section III, section IV presents a description about the data acquisition, data processing is describing in section $\mathrm{V}$, the implementation of the system is described in section VI, and the conclusions of this work is presented in section VII.

\section{RELATED WORK}

Huiyu Zhou and Huosheng $\mathrm{Hu}$ in [3] have used the accelerometer to implement a handy controlled robotic arm and they use the robotic arm to measure wrist and elbow positions with the aid of gyroscope.

Another handy controlled robotic arm in [4] has been implemented by N.Nandhini and SU.Suganthi to control the anthropomorphic robotic arm. They use accelerometers to measure the orientation and regular velocity of the lower arm. Their research presented a biomechatronic approach to the design of an anthropomorphic artificial hand.

In [5], Syed Aamair, Vuppula Manohar have develop a real time robotic arm using Micro-Electro-Mechanical Systems (MEMS). They control the directions of the robot using MEMS. According to the directions of MEMS like front, back, left and right robot is moved.

A mobile robotic arm was implemented by Vivek Bhojak, Girish Kumar Solanki and Sonu Daultani in [6]. A cheap and easy way of control using popular AVR microcontrollers and $\mathrm{RF}$ devices is implemented their research.

In [7], the researchers have developed a low power hand motion gesture technique which based on $3.3 \mathrm{~V}-5 \mathrm{~V}$ for the portable devices. Their design was developed by using MEMS sensor applications.

\section{PRELIMINARIES}

\subsection{Objective and Methodology}

The objective of the paper is to develop a robotic arm oriented by natural human arm movements whose data is acquired through the use of accelerometers, gyroscope and magneto sensors (MEMS Sensors). For proper control mechanism and to reduce the amount of noise coming in from the sensors, a proper averaging filter is used for smoothening the output of the accelerometer. The development of this arm is based on two Arduino platforms, one for signal processing and the other for decision making, the two boards are interfaced with each other using Bluetooth wireless communication.

The system in this research consists of two sides: sender side and receiver side. 
At sender side, Arduino board (Atmega328P) is used for acquiring sensing data and controlling process and to display the results (roll, Pitch and yaw angles of human arm movement) using LCD display. A flex resistor is also used, it is mounted on a glove, this resistor can sense finger movements to control the clamp of the robotic arm. A push bottom switch is used to control whether the (angles) are intended to move the Rover or the robotic arm then send the data via Bluetooth after encoding it.

On the receiving part, there is a Bluetooth device to receive the coded data, after decoding the incoming data, Arduino board (Atmega2560) translate those angles and check the status of the push bottom to either move the Rover or to control the robotic arm movements. The robot does not require any training process because it is fully controlled by the user hand movement. The hardware and software function are combined to make the system reliable.

\subsection{Prototyping Requirements}

The following are the hardware requirements that have been used to implement the prototype:

1. Arduino UNO Board Atmega238P (Data Acquisition).

2. Arduino Mega Atmega2560 (Controller).

3. Robotic Arm.

4. MEMS sensor contains accelerometers, gyroscope and magneto sensors all in one package.

5. Wireless Transmitter / Receiver (Bluetooth).

6. A predesigned robotic arm with servo motors.

7. Flex resistor (to control the clamp of the robot arm).

8. H-Bridge DC motor driver.

9. ROVER 5 robot platform.

10. Two DC motors.

11. Monitoring device (LCD).

\section{DATA ACQUISITION}

This section describes the process of getting the raw data from each sensor and demonstrates the functionality of each sensor. Arduino IDE version 1.6.8 was used to program the microcontroller, and to display data readings on $\mathrm{X}-\mathrm{Y}$ a new function called serial plot has been used.

\subsection{Accelerometers}

The readings of accelerometer are formulated as a vector in the device reference frame. This vector points down, towards the center of the earth, the device also capture accelerations. The accelerometer readings are noisy and the amount of noise depending on virtue of how the sensor works. Fig. 1 shows a plot of the raw data readings captured for 10 seconds.

\subsection{Gyroscopes}

Gyroscope measures the rotations, it returning an angular rotation vector also in the device reference frame. The gyro readings are very responsive to small amounts of movements. The main use of gyro sensor is calculating the position by keeping track of the current position and adjusting it every time-step, with every new gyro reading. An integration process must be used to convert gyroscope readings to an angle position, this process may introduce accumulated error with time. Fig.2 shows raw data readings of gyroscope captured for 10 seconds.

\subsection{Magnetometers}

Magnetometers measures magnetic fields around it, and it also giving a vector corresponding to the cumulative magnetic field. This sensor gives an estimated orientation of the device. This estimation is useful if readings of magnetometer are combined with the accelerometer sensor readings, because, the accelerometer sensor lack the information about the device yaws. Magnetometers are not affected by the earth magnetic field, but it can effect by any object with a magnetic field such as permanent magnets and also ferromagnetic metals, Fig. 3 shows the magnetometers readings captured for 10 seconds.

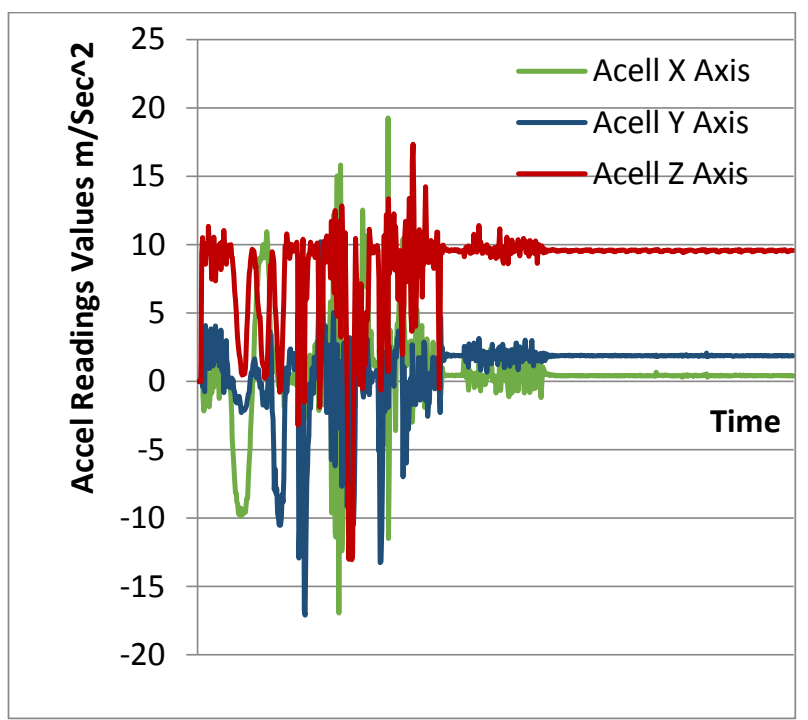

Fig.1 Raw MEMS Accelerometer Data

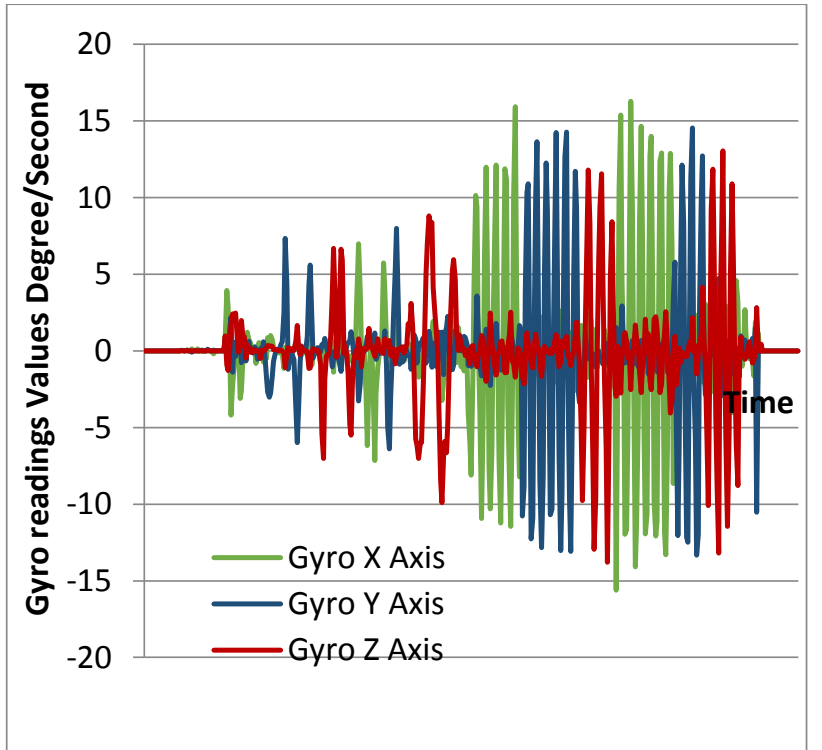

Fig.2 Raw MEMS Gyroscope Data (Movement Around 3Axis) 


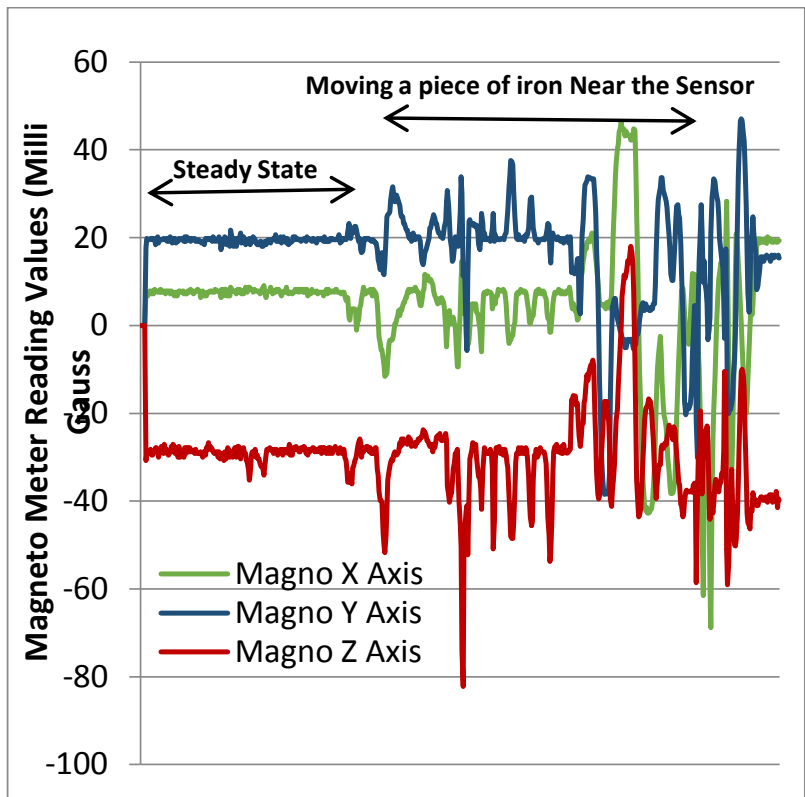

Fig.3 Raw MEMS Magnetometer Readings

\subsection{Raw Data Processing}

Sensor fusion technique must be used for converting the raw data to actual angles. Estimation of body orientation depending on single inertial sensor is not good idea, because each sensor has its strength and weakness. MEMS gyroscopes and accelerometer have numbers of error characteristics such as constant bias, thermo-Mechanical white noise / angle random walk, flicker noise / bias stability and calibration error $[8,9]$, also the accelerometers are very noisy and can never provide a yaw estimate. Magnetometers are affected by any magnetic field.

In order to estimate a correct body orientation, the three sensors may be used with each other via certain sensor fusion technique. The main idea behind sensor fusion is to combined readings from different sensors using one of sensor fusion algorithms such as complementary filter or Kalman filter to provide more useful results.

In this paper a complementary filter or balance filter has been used to get Pitch, Roll, and Yaw angles. This filter combine two filters with each other, one for the gyroscope and the other for the accelerometer to estimate pitch and roll, gyro and magneto for estimating yaw [10].

\section{DATA PROCESSING}

This section describes the operation of converting the raw data into real angles.

\subsection{Angle Detection of Gyroscope}

Since most of gyroscopes have bias errors [8], the drift errors occur from integration of the gyroscope readings. Fig. 4 shows this phenomenon. This error can be solved using a complementary filter described in section $\mathrm{C}$. The blue line in Fig. 4 is the angle obtained from the accelerometer, the orange line is the integration of the angular rates obtained from gyroscope and the red is the reading of the complementary filter output.

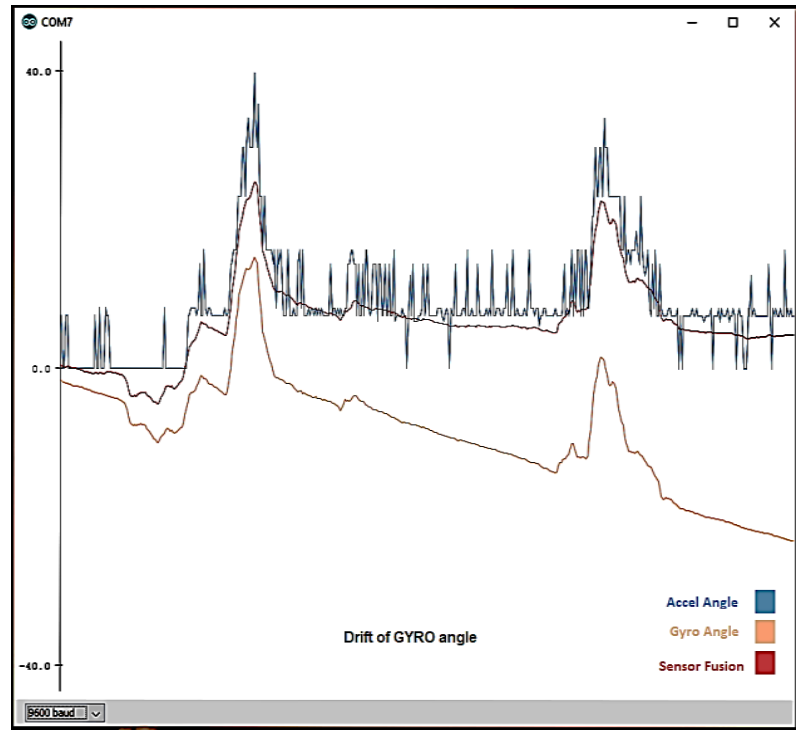

Fig.4 MEMS Gyro Data Drift

\subsection{Angle Detection of Accelerometer}

The amount of static acceleration due to the gravity can be measured using accelerometer. The tilled angle $(\theta c)$ with respect to the direction of the gravity can be found using this sensor. When the z- component $(\mathrm{Za})$ and $\mathrm{y}$ - component (Ya) of accelerometer is found. A roll angle $(\theta \mathrm{c})$ angle can be calculated using accelerometer readings using Eqn. 1 [10]. Fig. 5 shows the obtained angle $(\theta \mathrm{c})$ from MEMS accelerometer.

$$
\theta_{c}=\tan ^{-1}\left(\frac{Y_{a}}{Z_{a}}\right)
$$

The estimated angle using accelerometer is not stable [6]. Fig. (6) shows the angle obtained from the accelerometer and the angle after using sensor fusion. The blue plot is the angle obtained from accelerometer readings and the yellow plot is the angle after using the complementary filter. It is very obvious that accelerometer output is noisy with error.

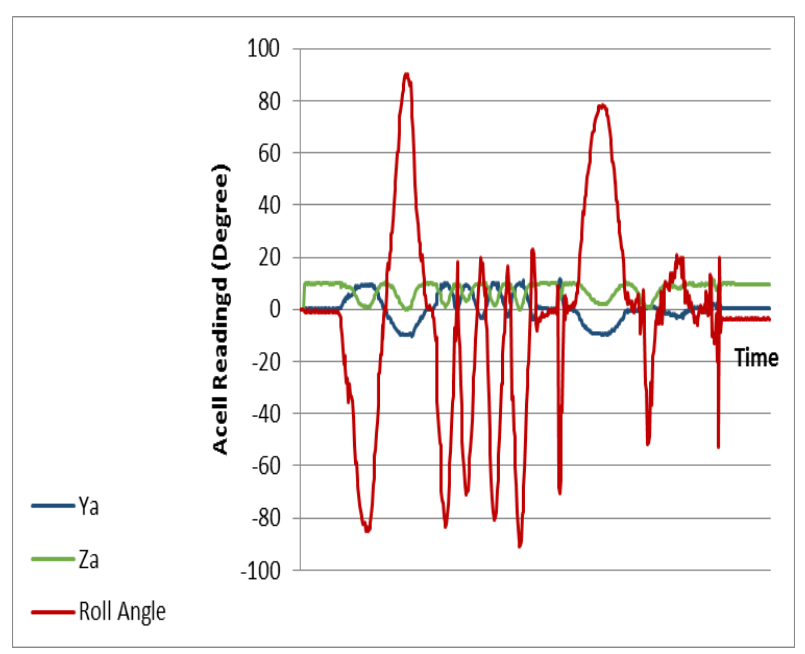

Fig.5 Angle Obtained from the MEMS Accelerometer Sensor 


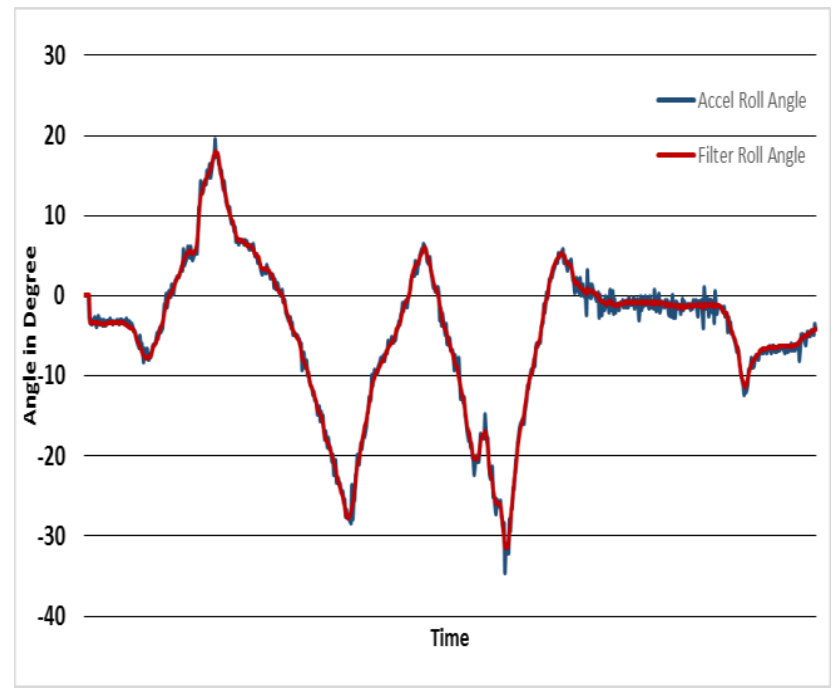

Fig. 6 Accelerometer output Vs. Complementary Output

\subsection{Complementary Filter}

Since a single MEMS gyro or accelerometer readings for body orientation estimation have a lack of accuracy; a complementary filter for data fusion must be used. The basic idea of the complementary filter is to combine the outputs of gyroscope and accelerometer. The estimate of the angle is obtained from the sum of their measurements. Fig.7 shows the structure of complementary filter [10].

The integration of the output of a gyroscope feeds into a high pass filter and the output of an accelerometer feeds into a low pass filter. And the sum of the high pass filter and the low pass filter must be 1 [8], that is:

$\mathrm{G}_{1}(\mathrm{~s})+\mathrm{G}_{2}(\mathrm{~s})=1$

According to Eqn.2, and to obtain accurate esitmation for hand orintation the following relationship of orientaion angle has been obtained experimentally:

Orientation Angle $=0.98$ (Last Angle+ Gyro Readings*Time step) +0.02 (Accelerometer Angle)

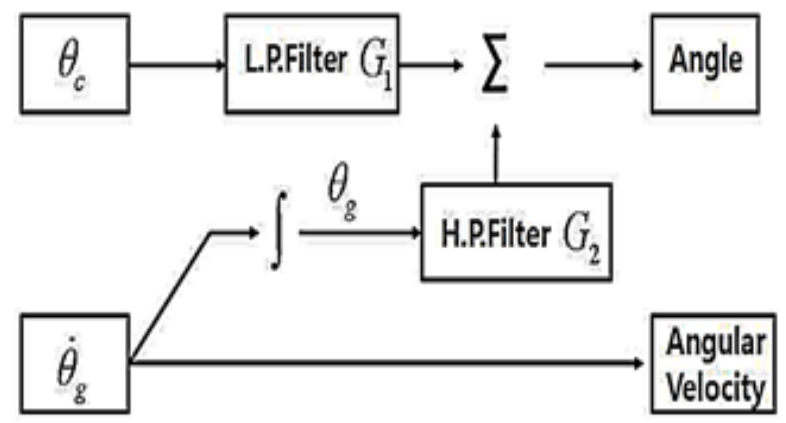

Fig.7 Structure of Complementary Filter

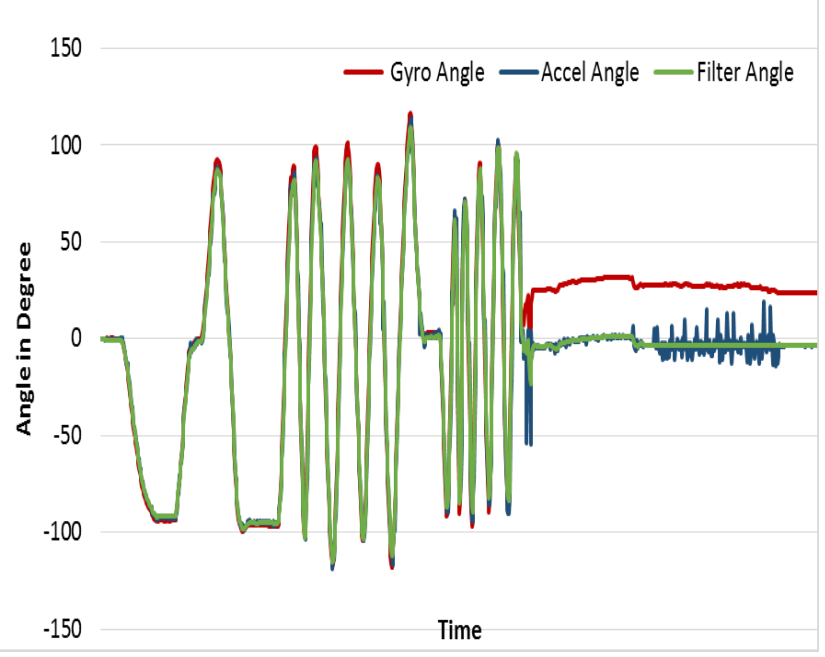

Fig.8 Complementary Filter Data Fusion Results

\section{SYSTEM IMPLEMENTATION 6.1 Implementation Steps}

The Hand motion controller designing and implementation steps are:

1. Designing the required software for reading of the raw data from the MEMS sensor.

2. Processing the data to get the right angles.

3. Adding a flex resistor to capture the finger movement to control the clamp.

4. Encoding the data to make it ready to transmit over Bluetooth

5. Add an LCD to display and monitor data.

A 3-axis gyroscope, 3-axis accelerometer and 3-axis digital compass are used and mounted on human hand using glove to work as gesture controller as shown in Fig. 9.while the robotic arm has been mounted over rover platform as shown in Fig. 10 .

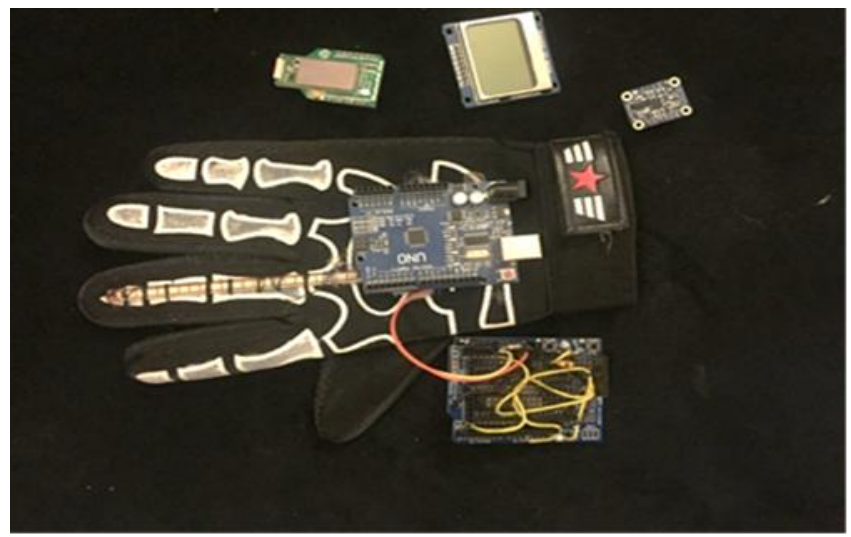

Fig. 9 Hand Motion Capture Device Components 


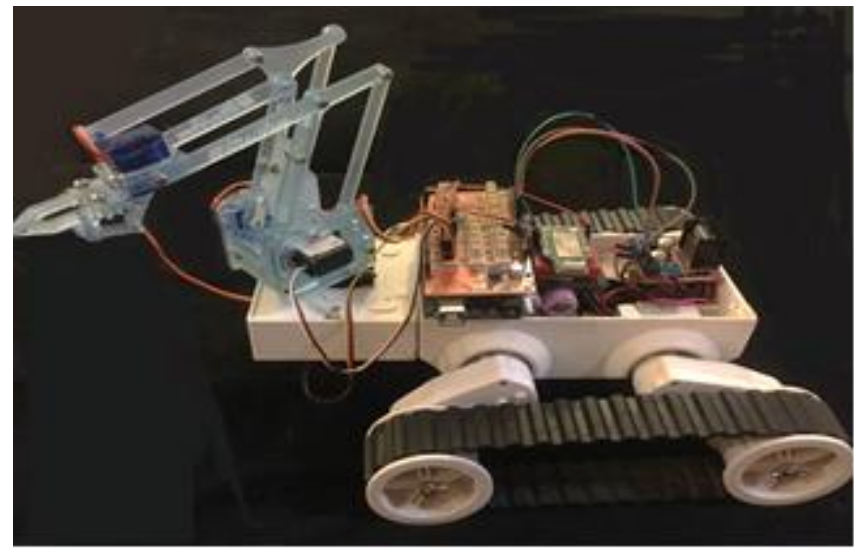

Fig. 10 Robotic Arm Mounted over the Rover Platform

\subsection{System Flow Chart}

All programs have been written using the Arduino IDE version 1.6.8. It's an open source compiler uses $\mathrm{C}$ language and it's also the programing software for all Arduino platforms. Fig. 11 shows the flow chart of the controller which fixed over human arm, while Fig. 12 shows the flow chart of robotic arm and rover platform controller.

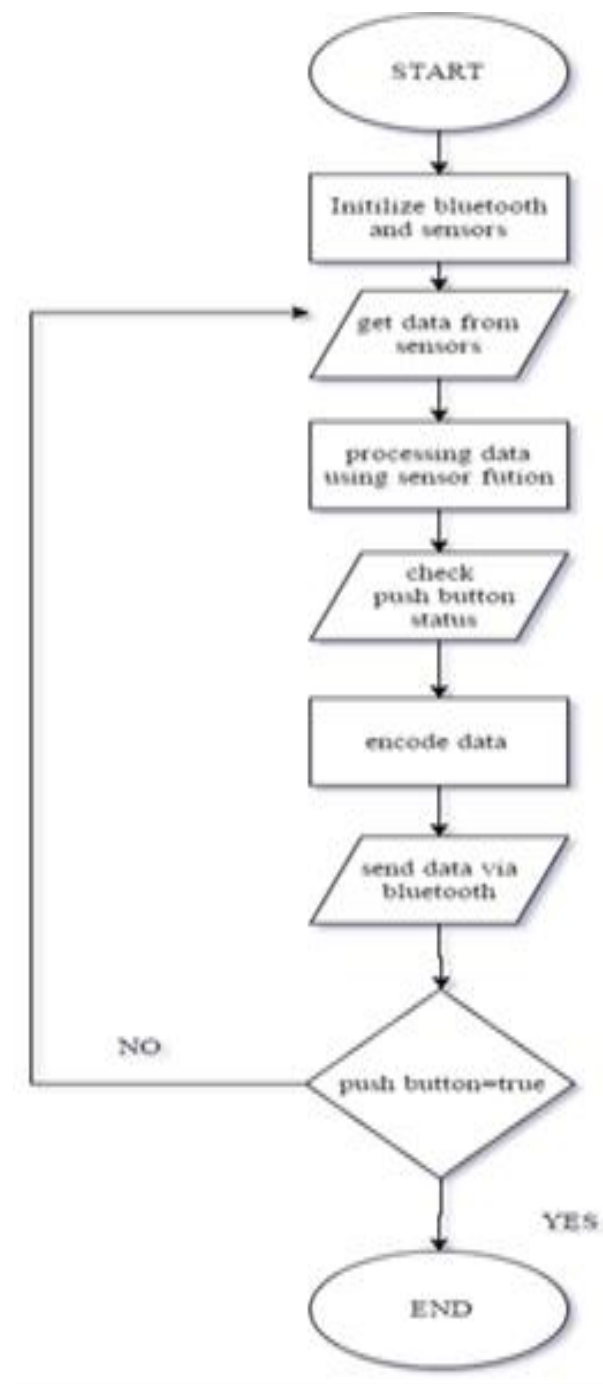

Fig. 11 Human Arm Motion Sensing Device

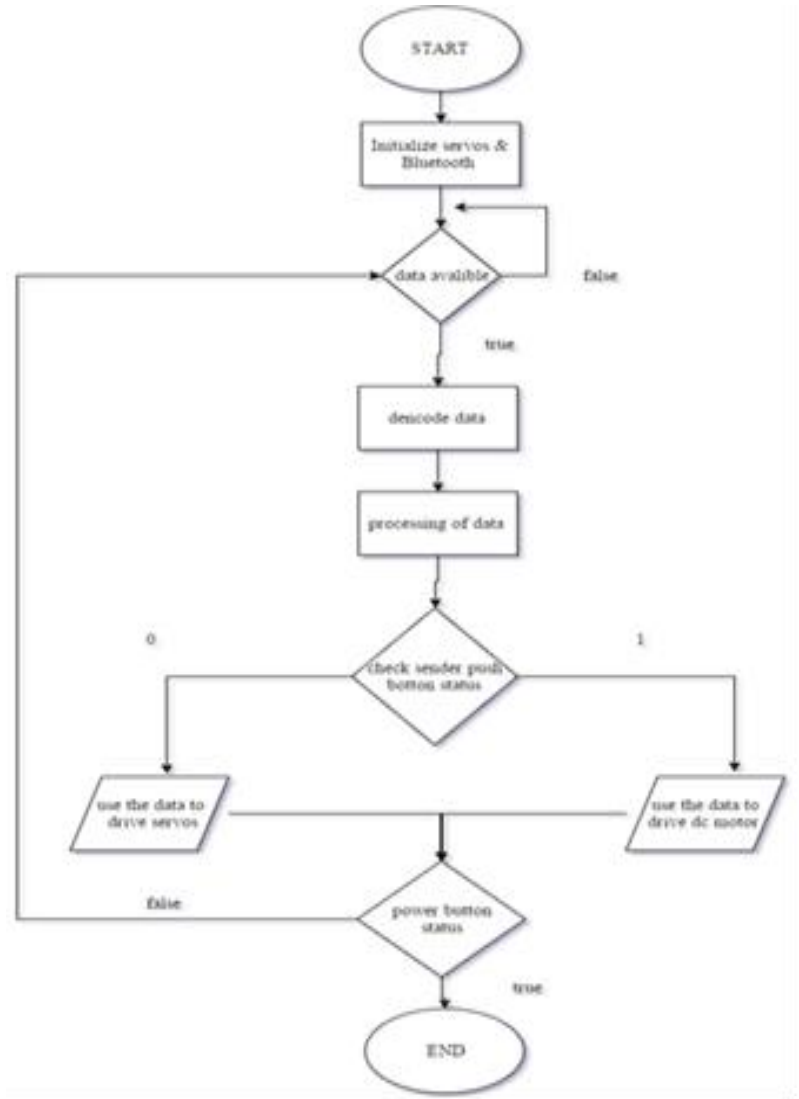

Fig. 12 Flow Chart of Robotic Arm and Rover Platform Controller

\section{CONCLUSIONS}

The research objectives have been achieved which were developing both hardware and software for controlling the robotic arm based on MEMS sensors. By reviewing the observations that have been completed, it obviously shows that the movements of robotic arm are accurate, easy control, specific, and it is friendly to use by the users. The robotic arm has been developed effectively such as the robot movements can be exactly controlled with the movements of human arm. The controlled method of the robotic arm presented in this paper is estimated to overcome many problems such as picking or placing objects that placed away from the users, pick and place dangerous objects in a very fast and easy way also, the arm presented in this paper can be used in medical application.

\section{REFERENCES}

[1] Love Aggarwal, Varnika Gaur and Puneet Verma,"Design and Implementation of a Wireless Gesture Controlled Robotic Arm with Vision," International Journal of Computer Applications Vol. 79 - No. 13, pp.39-43, October 2013.

[2] Hari Prabhat Gupta, Haresh S. Chudgar, Sidhartha Mukherjee, Tanima Dutta, and Kulwat Sharma, "A Continuous Hand Gestures Recognition Technique for Human-Machine Interaction Using Accelerometer and Gyroscope", IEEE Sensors Journal, Vol. 16, P.P 64256432, 2016.

[3] Huiyu Zhou and Huosheng Hu , "Reducing Drifts in the Inertial Measurements of Wrist and Elbow Positions," 
IEEE Transaction on Instrumentation and Measurement, Vol.59, Issue 3. pp. 575 - 585, Sep. 2009.

[4] N.Nandhini and SU.Suganthi " Wireless Control of a Robotic Arm Using Inertial Sensor," International Journal of Engineering and Advanced Technology, Vol.2, Issue 4, pp.318-320, April 2013.

[5] Syed Aamair, Vuppula Manohar, "Design and Development of Real Time Robotic ARM Control using ZIGBEE Wireless communication" International Journal of Professional Engineering Studies, Vol. 1, Issue 2, pp. 201-206, Dec. 2013.

[6] Vivek Bhojak, Girish Kumar Solanki and Sonu Daultani ," Gesture Controlled Mobile Robotic Arm Using Accelerometer" International Journal of Innovative Research in Science, Engineering and Technology, Vol. 4, Issue 6, pp. 4538-4544, Jun. 2015.
[7] G. Vijaya Kumar, Dr. Y. Padma SAI, V. Naveen Kumar, and A. Prathibha, "Hand Gesture Recognition Using Accelerometer for Disabled", International Journal of Science, Engineering and Technology Research (IJSETR), Vol. 3, Issue 12, 2014.

[8] Oliver J. Woodman, “An Introduction to Inertial Navigation, Technical Report" University of Cambridge, Aug. 2007.

[9] Meenaakumari.M and M.Muthulakshmi, "MEMS Accelerometer Based Hand Gesture Recognition", International Journal of Advanced Research in Computer Engineering \& Technology (IJARCET), Vol. 2, No. 5, 2013.

[10] Hyung Gi Min and Eun Tae Jeung, "Complementary Filter Design for Angle Estimation using MEMS Accelerometer and Gyroscope", Available online http://www.academia.edu/6261055. 